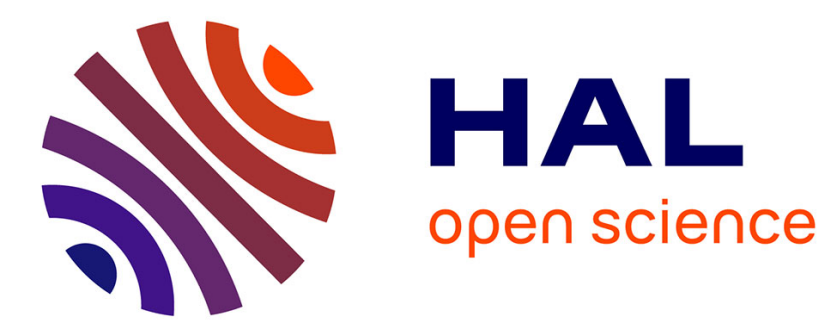

\title{
A PDE-ODE model for a junction with ramp buffer
}

Maria Laura Delle Monache, Samitha Samaranayake, Jack Reilly, Walid Krichene, Paola Goatin, Alexandre Bayen

\section{To cite this version:}

Maria Laura Delle Monache, Samitha Samaranayake, Jack Reilly, Walid Krichene, Paola Goatin, et al. A PDE-ODE model for a junction with ramp buffer. SIAM Journal on Applied Mathematics, 2014, 74 (1), pp.22-39. hal-00786002

\section{HAL Id: hal-00786002 https://hal.inria.fr/hal-00786002}

Submitted on 7 Feb 2013

HAL is a multi-disciplinary open access archive for the deposit and dissemination of scientific research documents, whether they are published or not. The documents may come from teaching and research institutions in France or abroad, or from public or private research centers.
L'archive ouverte pluridisciplinaire HAL, est destinée au dépôt et à la diffusion de documents scientifiques de niveau recherche, publiés ou non, émanant des établissements d'enseignement et de recherche français ou étrangers, des laboratoires publics ou privés. 


\title{
A PDE-ODE MODEL FOR A JUNCTION WITH RAMP BUFFER
}

\author{
M. L. DELlE MONACHE ${ }^{\dagger} *$, J. REILLY ${ }^{\ddagger}$, S. SAMARANAYAKE $\ddagger \S$, W. KRICHENE $\ddagger \S$, P. \\ GOATIN $^{\dagger}$, AND A. M. BAYEN ${ }^{\ddagger}$
}

\begin{abstract}
We consider the Lighthill-Witham-Richards traffic flow model on a junction composed by one mainline, an onramp and an offramp, which are connected by a node. The onramp dynamics is modeled using an ordinary differential equation describing the evolution of the queue length. The definition of the solution of the Riemann problem at the junction is based on an optimization problem and the use of a right-of-way parameter. The numerical approximation is carried out using Godunov scheme, modified to take into account the effects of the onramp buffer. We present the result of some simulations and check numerically the convergence of the method.
\end{abstract}

Key words. Scalar conservation laws, PDE-ODE systems, Riemann problem, Macroscopic traffic flow models

AMS subject classifications. 90B20, 35L65

1. Introduction. Hydrodynamic models have commonly been used in the literature to describe the macroscopic evolution of vehicular traffic on roads and have been successfully generalized to networks in recent years. In the 1950s, Lighthill and Whitham 18 and Richards 21, independently, proposed a fluid dynamic model for traffic flow on an infinite single road, using a non-linear hyperbolic partial differential equation (PDE). The Cauchy problem has successfully been extended to initialboundary value problem in [1] and then developed specifically for scalar conservation laws with concave flux in [15], and for traffic applications in 22. More recently, several authors proposed models on networks that take into account different type of solutions at the intersections, see [4, 5, 7, 8, 11, 12 and the references therein.

In this article, we focus on a junction model designed for a ramp metering problem. Ramp metering models have been introduced in the engineering community in a discrete setting, see [19, 20] for details. In this article, we apply a continuous approach. We consider the scalar Lighthill-Whitham-Richards model on a network composed of a single junction connecting a mainline, an onramp and an offramp. The mainline evolution is described by a scalar conservation law, while the onramp dynamics is modeled by a buffer of infinite capacity, which is defined by an ordinary differential equation (ODE) depending on the difference between the incoming and outgoing fluxes at the ramp.

In the following sections, we prove the existence and uniqueness of solutions of the Riemann problem at the junction. The results are obtained by solving a Linear Programming $(L P)$ optimization problem. Unlike [9, where the flux through the junction is maximized, our $L P$-optimization consists in maximizing the flux on the outgoing mainline, see Remark 3 below. The offramp is treated as a sink, and a priority parameter is introduced to ensure uniqueness of the solution. As a modeling choice, the priority is satisfied in an approximate way, i.e., the priority will not always be respected, in benefit of flux maximization.

\footnotetext{
*Email: maria-laura.delle_monache@inria.fr, Corresponding author.

${ }^{\dagger}$ Inria Sophia Antipolis - Méditerranée, Sophia Antipolis, France

${ }^{\ddagger}$ University of California, Berkeley, USA

$\S$ The authors have been supported by the ERC Starting Grant 2010 under the project 'TRAffic Management by Macroscopic Models", the INRIA associated team 'Optimal REroute Strategies for Traffic managEment" and the France-Berkeley Fund under the project "Optimal Traffic Flow Management with GPS Enabled Smartphones".
} 
The article presents numerical approximations of possibly discontinuous solutions obtained using this model. In particular, we suitably modify the Godunov scheme to include the boundary conditions at the junction, as in [3], and the ODE describing the buffer. This allows one to take into account the possible creation of an additional shock when the buffer empties. The scheme provides accurate numerical approximations, as shown by the numerical tests provided here.

The article is organized as follows. Section 2 contains some preliminary notations and definitions, while $\$ 3$ describes in details the solution of the Riemann problem at the junction. In $\$$ we introduce the numerical scheme with the particular boundary conditions used to compute approximate solutions to the problem. In $\$ 5$ we present some numerical tests which show the effectiveness of our approximation. Throughout the paper, we refer to [2, 6] for the general theory of hyperbolic conservation laws and to [16, 17] for an introduction to the main numerical concepts.

2. Fundamental Definitions and Notations. We consider a junction with one mainline $I$ modeled by the real line ] $-\infty,+\infty$ [, one onramp $R_{1}$ and one offramp $R_{2}$ at $x=0$, as illustrated in Figure 2.1.

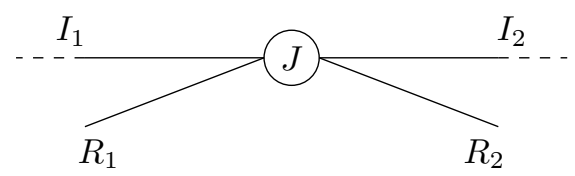

Fig. 2.1: Junction modeled in the article.

From a macroscopic point of view, this means that on each mainline segment $\left.I_{1}=\right]-\infty, 0\left[\right.$ and $\left.I_{2}=\right] 0,+\infty[$, we consider the mass conservation equation:

$$
\partial_{t} \rho+\partial_{x} f(\rho)=0, \quad(t, x) \in \mathbb{R}^{+} \times I_{i},
$$

where $\rho=\rho(t, x) \in\left[0, \rho_{\max }\right]$ is the mean traffic density, $\rho_{\max }$ is the maximal density allowed on the road and the flux function $f:\left[0, \rho_{\max }\right] \rightarrow \mathbb{R}^{+}$is given by the following flux-density relation

$$
f(\rho)=\rho v(\rho)
$$

where $v(\rho)$ is a smooth decreasing function denoting the mean traffic speed. Throughout the article, we assume for simplicity that:

(A1) $\rho_{\max }=1$

(A2) $f(0)=f(1)=0$;

(A3) $f$ is a strictly concave function.

Assumptions (A2) and (A3) ensure existence and uniqueness of a point of maximum of the flux function $\rho^{\text {cr }} \in[0,1]$. A typical example of flux function for the LWR model is given in Figure 2.2 . 


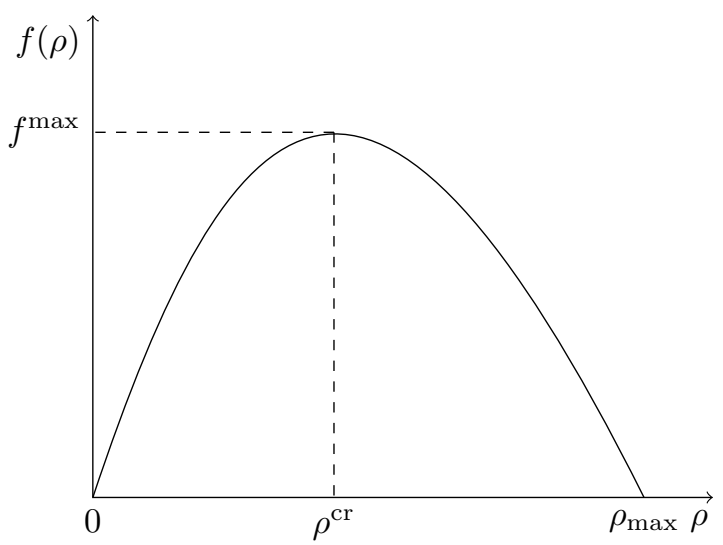

Fig. 2.2: Flux function of equation (2.1), commonly referred to as fundamental diagram in the transportation literature.

On the onramp $R_{1}$ we consider the presence of a buffer modeled by the following ODE:

$$
\frac{d l(t)}{d t}=F_{\mathrm{in}}(t)-\gamma_{\mathrm{r} 1}(t), \quad t \in \mathbb{R}^{+},
$$

where $l(t) \in\left[0,+\infty\left[\right.\right.$ is the length of the queue, $F_{\text {in }}(t)$ is the flux that enters the onramp and $\gamma_{\mathrm{r} 1}(t)$ is the flux that exits from the onramp.

This particular choice is taken to avoid backward waves on the onramp boundary, which happens in the case of horizontal queues. In particular, at the left boundary of the onramp, backward moving shock waves can result in lost information on the flux that actually enters the buffer. The presence of the buffer helps accounting for all the flow that enters the onramp, for details see 13. We consider the offramp as an infinite sink that accepts all the flux entering from the mainline $I_{1}$, and we assume that no flux from the onramp is allowed in the offramp.

The Cauchy problem to solve is then:

$$
\begin{cases}\partial_{t} \rho_{i}+\partial_{x} f\left(\rho_{i}\right)=0, & (t, x) \in \mathbb{R}^{+} \times I_{i}, i=1,2 \\ \frac{d l(t)}{d t}=F_{\mathrm{in}}(t)-\gamma_{\mathrm{r} 1}(t), & t \in \mathbb{R}^{+}, \\ \rho_{i}(0, x)=\rho_{i, 0}(x), & \text { on } I_{i} i=1,2 \\ l(0)=l_{0}, & \end{cases}
$$

where $\rho_{i}(0, x)$ represents the initial condition and $l_{0} \in[0,+\infty[$ is the initial load of the buffer.

This will be coupled with an optimization problem at the junction which will give the distribution of the traffic among the roads.

We define the demand $d\left(F_{\text {in }}, l\right)$ of the onramp, the demand function $\delta\left(\rho_{1}\right)$ on the incoming mainline segment corresponding to the density $\rho_{1}$, and the supply function 
$\sigma\left(\rho_{2}\right)$ on the outgoing mainline segment corresponding to the density $\rho_{2}$ as follows.

$$
\begin{aligned}
d\left(F_{\mathrm{in}}, l\right) & = \begin{cases}\gamma_{\mathrm{r} 1}^{\max } & \text { if } l(t)>0, \\
\min \left(F_{\mathrm{in}}(t), \gamma_{\mathrm{r} 1}^{\max }\right) & \text { if } l(t)=0,\end{cases} \\
\delta\left(\rho_{1}\right) & = \begin{cases}f\left(\rho_{1}\right) & \text { if } 0 \leq \rho_{1}<\rho^{\mathrm{cr}}, \\
f^{\max } & \text { if } \rho^{\mathrm{cr}} \leq \rho_{1} \leq 1,\end{cases} \\
\sigma\left(\rho_{2}\right) & = \begin{cases}f^{\max } & \text { if } 0 \leq \rho_{2} \leq \rho^{\mathrm{cr}}, \\
f\left(\rho_{2}\right) & \text { if } \rho^{\mathrm{cr}}<\rho_{2} \leq 1,\end{cases}
\end{aligned}
$$

where $\gamma_{\mathrm{r} 1}^{\max }$ is the maximal flow on the onramp and $f^{\max }=f\left(\rho^{\mathrm{cr}}\right)$ is the maximal flux on $I_{1}$ and $I_{2}$. Moreover, we introduce $\beta \in[0,1]$ the split ratio of the offramp, and $\gamma_{\mathrm{r} 2}(t)=\beta f\left(\rho_{1}(t, 0-)\right)$ its flux.

Definition 2.1. A triple $\left(\rho_{1}, \rho_{2}, l\right) \in \prod_{i=1}^{2} \mathcal{C}^{0}\left(\mathbb{R}^{+} ; \mathbf{L}^{\mathbf{1}} \cap B V(\mathbb{R})\right) \times \mathbf{W}^{\mathbf{1}, \infty}\left(\mathbb{R}^{+} ; \mathbb{R}^{+}\right)$ is an admissible solution to (2.3) if

(i) $\rho_{1}, \rho_{2}$ are weak solutions on $I_{1}, I_{2}$, i.e., $\rho_{i}:\left[0,+\infty\left[\times I_{i} \rightarrow[0,1], i=1,2\right.\right.$, such that

$$
\int_{\mathbb{R}^{+}} \int_{I_{i}}\left(\rho_{i} \partial_{t} \varphi_{i}+f\left(\rho_{i}\right) \partial_{x} \varphi_{i}\right) d x d t=0, \quad i=1,2,
$$

for every $\varphi_{i} \in \mathcal{C}_{c}^{1}\left(\mathbb{R}^{+} \times I_{i}\right)$.

(ii) $\rho_{i}$ satisfies the Kružhkov entropy condition [14] on $\left(\mathbb{R} \times I_{i}\right)$, i.e., for every $k \in[0,1]$ and for all $\varphi_{i} \in \mathcal{C}_{c}^{1}\left(\mathbb{R}^{+} \times I_{i}\right), t>0$,

$$
\begin{gathered}
\int_{\mathbb{R}^{+}} \int_{I_{i}}\left(\left|\rho_{i}-k\right| \partial_{t} \varphi_{i}+\operatorname{sgn}\left(\rho_{i}-k\right)\left(f\left(\rho_{i}\right)-f(k)\right) \partial_{x} \varphi_{i}\right) d x d t \\
+\int_{I_{i}}\left|\rho_{i, 0}-k\right| \varphi_{i}(0, x) d x \geq 0 ; \quad i=1,2 .
\end{gathered}
$$

(iii) $f\left(\rho_{1}(t, 0-)\right)+\gamma_{\mathrm{r} 1}(t)=f\left(\rho_{2}(t, 0+)\right)+\gamma_{\mathrm{r} 2}(t)$.

(iv) The flux of the outgoing mainline $f\left(\rho_{2}(t, 0+)\right)$ is maximum subject to

$$
f\left(\rho_{2}(t, 0+)\right)=\min \left((1-\beta) \delta\left(\rho_{1}(t, 0-)\right)+d\left(F_{\text {in }}(t), l(t)\right), \sigma\left(\rho_{2}(t, 0+)\right)\right),
$$

and (iii)

(v) $l$ is a solution of $(2.2)$ for a.e. $t \in \mathbb{R}^{+}$.

REMARK 1. A parameter $P$ is introduced to ensure uniqueness of the solution. $P \in] 0,1[$ is a right of way parameter that defines the amount of flux that enters the outgoing road from each incoming road. In particular, $P f\left(\rho_{1}(t, 0-)\right)$ is the flux allowed from the incoming mainline into the outgoing mainline, and $(1-P) \gamma_{\mathrm{r} 1}(t)$ the flux from the onramp.

3. Riemann problem at the junction. In this section, we construct step by step the Riemann Solver at the junction. We fix constants $\rho_{1,0}, \rho_{2,0} \in[0,1]$, $l_{0} \in\left[0,+\infty\left[, F_{\text {in }} \in\right] 0,+\infty[\right.$ and a priority factor $P \in] 0,1[$. The Riemann problem at $J$ is the Cauchy problem 2.3 where the initial conditions are given by $\rho_{0, i}(x) \equiv \rho_{0, i}$ in $I_{i}$ for $i=1,2$. We define the Riemann Solver by means of a Riemann Solver $\mathcal{R S}_{\bar{l}}$, which depends on the instantaneous load of the buffer $\bar{l}$. For each $\bar{l}$ the Riemann Solver $\mathcal{R S}_{\bar{l}}$ is constructed in the following way. 
1. Define $\Gamma_{1}=f\left(\rho_{1}(t, 0-)\right), \Gamma_{2}=f\left(\rho_{2}(t, 0+)\right), \Gamma_{\mathrm{r} 1}=\gamma_{\mathrm{r} 1}(t)$;

2. Consider the space $\left(\Gamma_{1}, \Gamma_{\mathrm{r} 1}\right)$ and the sets $\mathcal{O}_{1}=\left[0, \delta\left(\rho_{1}\right)\right], \mathcal{O}_{\mathrm{r} 1}=\left[0, d\left(F_{\mathrm{in}}, \bar{l}\right)\right]$;

3. Trace the lines $(1-\beta) \Gamma_{1}+\Gamma_{\mathrm{r} 1}=\Gamma_{2}$; and $\Gamma_{1}=\frac{P}{1-P} \Gamma_{\mathrm{r} 1}$;

4. Consider the region

$$
\Omega=\left\{\left(\Gamma_{1}, \Gamma_{\mathrm{r} 1}\right) \in \mathcal{O}_{1} \times \mathcal{O}_{\mathrm{r} 1}:(1-\beta) \Gamma_{1}+\Gamma_{\mathrm{r} 1} \in\left[0, \Gamma_{2}\right]\right\} .
$$

Different situations can occur depending on the value of $\Gamma_{2}$ :

(i) Demand limited case: $\Gamma_{2}=(1-\beta) \delta\left(\rho_{1}(t, 0-)\right)+d\left(F_{\text {in }}, \bar{l}\right)$.

We set $Q$ to be the point $\left(\hat{\Gamma}_{1}, \hat{\Gamma}_{\mathrm{r} 1}\right)$ such that $\hat{\Gamma}_{1}=\delta\left(\rho_{1}(t, 0-)\right), \hat{\Gamma}_{\mathrm{r} 1}=d\left(F_{\mathrm{in}}, \bar{l}\right)$ and $\hat{\Gamma}_{2}=(1-\beta) \delta\left(\rho_{1}(t, 0-)\right)+d\left(F_{\text {in }}, \bar{l}\right)$, as illustrated in Figure 3.1 (a).

(ii) Supply limited case: $\Gamma_{2}=\sigma\left(\rho_{2}(t, 0+)\right)$.

We set $Q$ to be the point of intersection of $(1-\beta) \Gamma_{1}+\Gamma_{\mathrm{r} 1}=\Gamma_{2}$ and $\Gamma_{1}=\frac{P}{1-P} \Gamma_{\mathrm{r} 1}$. If $Q \in \Omega$, we set $\left(\hat{\Gamma}_{1}, \hat{\Gamma}_{\mathrm{r} 1}\right)=Q$ and $\hat{\Gamma}_{2}=\Gamma_{2}$, see Figure 3.1.(b) if $Q \notin \Omega$, we set $\left(\hat{\Gamma}_{1}, \hat{\Gamma}_{\mathrm{r} 1}\right)=S$ and $\hat{\Gamma}_{2}=\Gamma_{2}$, where $S$ is the point of the segment $\Omega \cap\left(\Gamma_{1}, \Gamma_{\mathrm{r} 1}\right):(1-\beta) \Gamma_{1}+\Gamma_{\mathrm{r} 1}=\Gamma_{2}$ closest to the line $\Gamma_{1}=\frac{P}{1-P} \Gamma_{\mathrm{r} 1}$, obtained solving the problem

$$
\begin{array}{rr}
\text { minimize } & \left\|\left(\begin{array}{c}
\gamma_{\mathrm{r} 1}(t) \\
f\left(\rho_{1}(t, 0-)\right)
\end{array}\right)-\left[\left(\begin{array}{c}
\gamma_{\mathrm{r} 1}(t) \\
f\left(\rho_{1}(t, 0-)\right)
\end{array}\right) \cdot \alpha^{P}\right] \alpha^{P}\right\|_{2}^{2} \\
\text { subject to } & f\left(\rho_{2}(t, 0+)\right)=(1-\beta) f\left(\rho_{1}(t, 0-)\right)+\gamma_{\mathrm{r} 1}(t), \\
\gamma_{\mathrm{r} 1}(t) \leq d\left(F_{\mathrm{in}}, \bar{l}\right), \\
f\left(\rho_{1}(t, 0+)\right) \leq \delta\left(\rho_{1}\right),
\end{array}
$$

where $\alpha^{P}$ is the normalized vector $\alpha^{P}=\frac{1}{\sqrt{P^{2}+(1-P)^{2}}}\left(\begin{array}{c}P \\ 1-P\end{array}\right)$, see Fig 3.1 (c)

As can be seen in Figure 3.1 (c), it might not be possible to respect the priority given by the parameter $P$ if we want to maximize also the flux.

Once we have determined $\hat{\Gamma}_{1}$ and $\hat{\Gamma}_{2}$, we can define $\hat{\rho}_{1}, \hat{\rho}_{2}$ in a unique way as follows. We recall that $\left.\rho=\rho^{\text {cr }} \in\right] 0,1[$ is the unique point of maximum of the flux and we define the function $\tau$ as follows, for details see 9 .

Definition 3.1. Let $\tau:[0,1] \rightarrow[0,1]$ be the map such that:

(i) $f(\tau(\rho))=f(\rho)$ for every $\rho \in[0,1]$;

(ii) $\tau(\rho) \neq \rho$ for every $\rho \in[0,1] \backslash\left\{\rho^{\text {cr }}\right\}$.

Given

$$
\rho_{1}(0, \cdot) \equiv \rho_{1,0}, \rho_{2}(0, \cdot) \equiv \rho_{2,0},
$$

there exists a unique couple $\left(\hat{\rho}_{1}, \hat{\rho}_{2}\right) \in[0,1]^{2}$ such that

$$
\hat{\rho}_{1} \in\left\{\begin{array}{ll}
\left.\left.\left\{\rho_{1,0}\right\} \cup\right] \tau\left(\rho_{1,0}\right), 1\right] & \text { if } 0 \leq \rho_{1,0} \leq \rho^{\text {cr }}, \\
{\left[\rho^{\text {cr }}, 1\right]} & \text { if } \rho^{\mathrm{cr}} \leq \rho_{1,0} \leq 1 ;
\end{array} \quad f\left(\hat{\rho}_{1}\right)=\hat{\Gamma}_{1},\right.
$$

and

$$
\hat{\rho}_{2} \in\left\{\begin{array}{ll}
{\left[0, \rho^{\mathrm{cr}}\right]} & \text { if } 0 \leq \rho_{2,0} \leq \rho^{\mathrm{cr}}, \\
\left\{\rho_{2,0}\right\} \cup\left[0, \tau\left(\rho_{2,0}\right)[\right. & \text { if } \rho^{\mathrm{cr}} \leq \rho_{1,0} \leq 1 ;
\end{array} \quad f\left(\hat{\rho}_{2}\right)=\hat{\Gamma}_{2} .\right.
$$




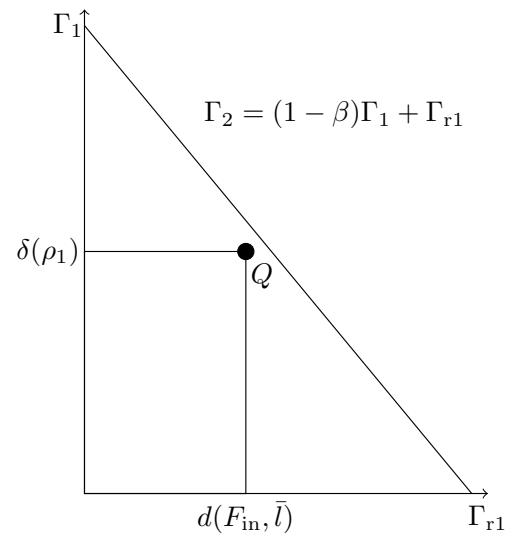

(a) Demand limited case

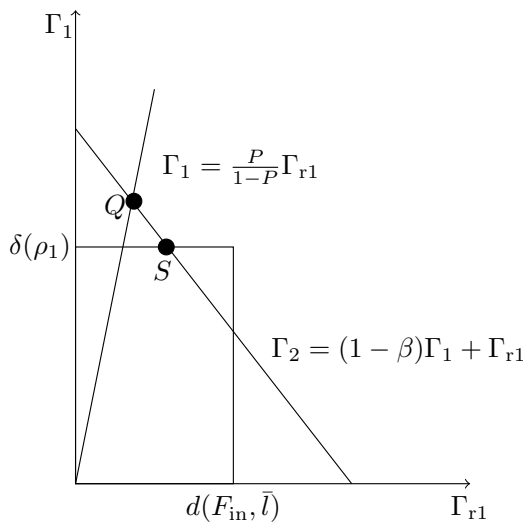

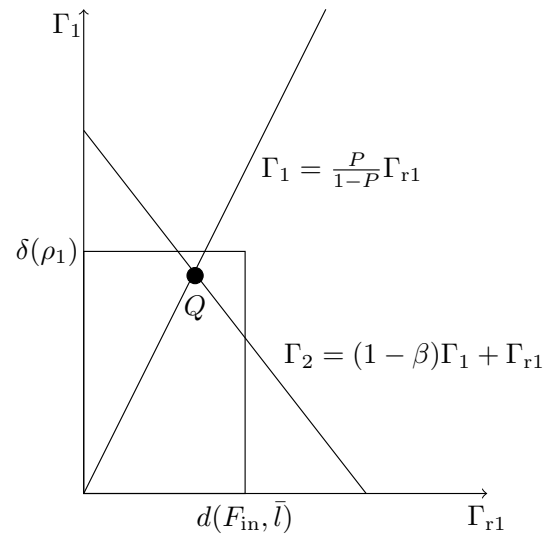

(b) Supply limited case: intersection inside $\Omega$

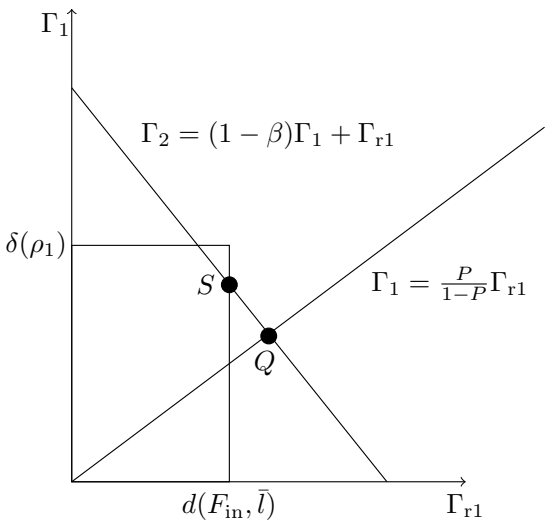

(c) Supply limited case: intersection outside $\Omega$

Fig. 3.1: Solutions of the Riemann Solver at the junction.

For the incoming road the solution is given by the wave $\left(\rho_{1,0}, \hat{\rho}_{1}\right)$, while for the outgoing road the solution is given by the wave $\left(\hat{\rho}_{2}, \rho_{2,0}\right)$. In this setting, given any initial data $\rho_{1,0}, \rho_{2,0}$, we can define $\mathcal{R S}_{\bar{l}}:[0,1]^{2} \rightarrow[0,1]^{2}$ by

$$
\mathcal{R S}_{\bar{l}}\left(\rho_{1,0}, \rho_{2,0}\right)=\left(\hat{\rho}_{1}, \hat{\rho}_{2}\right) \text {. }
$$

Now given the initial load of the buffer $l_{0}=\bar{l}$, the function $l(t)$ at time $t>0$ is given according to the following possibilities, determined by straight integration of 2.2 :

1. If $F_{\text {in }}<\hat{\Gamma}_{\text {r1 }}$, then

$$
l(t)= \begin{cases}l_{0}+\left(F_{\mathrm{in}}-\hat{\Gamma}_{\mathrm{r} 1}\right) t & \text { if } 0<t<\frac{l_{0}}{\hat{\Gamma}_{\mathrm{r} 1}-F_{\mathrm{in}}}, \\ 0 & \text { if } t>\frac{l_{\Gamma_{\mathrm{r} 1}-F_{\mathrm{in}}}}{}\end{cases}
$$

2. If $F_{\text {in }} \geq \hat{\Gamma}_{\mathrm{r} 1}$, then

$$
l(t)=l_{0}+\left(F_{\text {in }}-\hat{\Gamma}_{\mathrm{r} 1}\right) t \quad \forall t>0 .
$$


REMARK 2. The presence of the buffer can create waves at time $\bar{t}>0$. In particular, we have new waves at time $\bar{t}=-l_{0} /\left(F_{\mathrm{in}}-\hat{\Gamma}_{\mathrm{r} 1}\right)$ (with new values of the densities $\left.\bar{\rho}_{1}, \bar{\rho}_{2}\right)$ if $F_{\mathrm{in}}<\hat{\Gamma}_{\mathrm{r} 1}$ and the buffer empties, see Figure 3.2.

No waves are created instead if $F_{\mathrm{in}} \geq \hat{\Gamma}_{\mathrm{r} 1}$, due to the infinity capacity of the buffer.

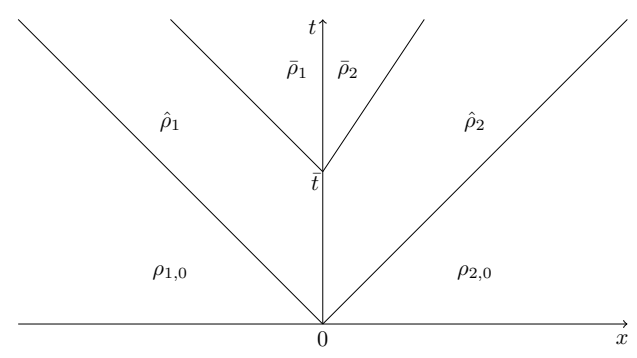

Fig. 3.2: Solution of the Riemann Problem.

The following theorem ensures consistency of $\mathcal{R S}_{\bar{l}}$.

TheOREm 3.2. Consider a junction $J$ and fix a priority parameter $P \in] 0,1[$. For every $\rho_{1,0}, \rho_{2,0} \in[0,1]$ and $l_{0} \in[0,+\infty[$, there exists a unique admissible solution $\left(\rho_{1}(t, x), \rho_{2}(t, x), l(t)\right)$ in the sense of Definition 2.1 satisfying the priority (possibly in an approximate way). Moreover, for a.e. $t>0$, it holds

$$
\left(\rho_{1}(t, 0-), \rho_{2}(t, 0+)\right)=\mathcal{R S}_{l(t)}\left(\rho_{1}(t, 0-), \rho_{2}(t, 0+)\right) .
$$

The proof of the theorem is deferred after some preliminary results. The mapping $\tau(\rho)$ as defined in (3.1) and the functions $\delta\left(\rho_{1}\right), d\left(F_{\text {in }}, l\right)$ and $\sigma\left(\rho_{2}\right)$ yield the following properties.

LEMma 3.3. If $\left(\hat{\rho}_{1}, \hat{\rho}_{2}\right)$ is a solution of the Riemann problem with initial data $\left(\rho_{1,0}, \rho_{2,0}\right)$, then the following holds:

$$
\begin{aligned}
\delta\left(\rho_{1,0}\right) & \leq \delta\left(\hat{\rho}_{1}\right), \\
\sigma\left(\rho_{2,0}\right) & \leq \sigma\left(\hat{\rho}_{2}\right), \\
d\left(F_{\text {in }}, l_{0}\right) & \leq d\left(F_{\text {in }}, l\right) .
\end{aligned}
$$

Proof. For the incoming road it holds:

$$
\begin{aligned}
& \delta\left(\rho_{1,0}\right) \leq \delta\left(\hat{\rho}_{1}\right) \quad \text { if } 0 \leq \rho_{1,0} \leq \rho^{\mathrm{cr}} \\
& \delta\left(\rho_{1,0}\right)=\delta\left(\hat{\rho}_{1}\right) \quad \text { if } \rho^{\mathrm{cr}} \leq \rho_{1,0} \leq 1 .
\end{aligned}
$$

In particular, if $\rho_{1,0} \in\left[0, \rho^{\text {cr }}\right]$, either $\left.\left.\hat{\rho}_{1} \in\right] \tau\left(\rho_{1,0}\right), 1\right]$ or $\hat{\rho}_{1}=\rho_{1,0}$. In the first case, $\delta\left(\rho_{1,0}\right)=f\left(\rho_{1,0}\right) \leq f^{\max }=\delta\left(\hat{\rho}_{1}\right)$, see Figure 3.3(a), while in the second case $\delta\left(\rho_{1,0}\right)=f\left(\rho_{1,0}\right)=f\left(\hat{\rho}_{1}\right)=\delta\left(\hat{\rho}_{1}\right)$, see Figure 3.3(b) On the other hand, if $\rho_{1,0} \in\left[\rho^{\text {cr }}, 1\right]$ then $\hat{\rho}_{1} \in\left[\rho^{\text {cr }}, 1\right]$ and $\delta\left(\rho_{1,0}\right)=f^{\max }=\delta\left(\hat{\rho}_{1}\right)$, see Figure 3.3( (c) . 


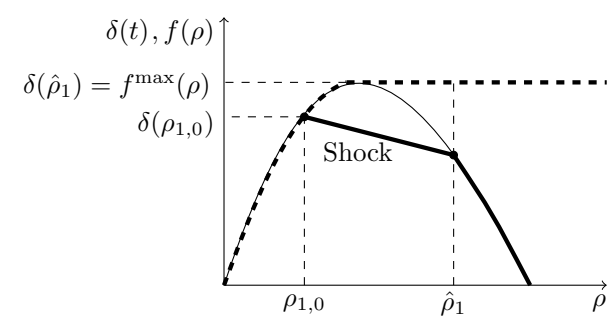

(a) $0 \leq \rho_{1,0} \leq \rho^{\text {cr }}$

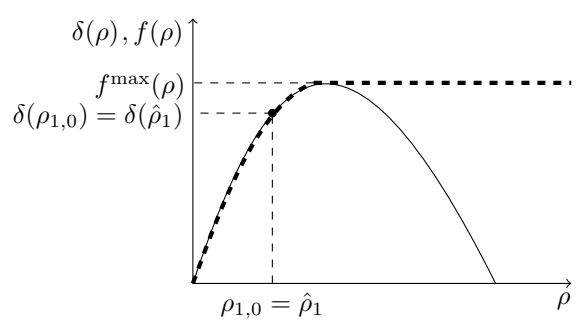

(b) $\rho_{1,0}=\hat{\rho}_{1}$

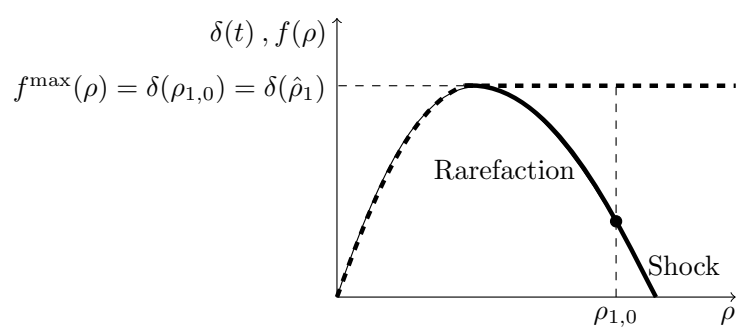

(c) $\rho^{\mathrm{cr}} \leq \rho_{1,0} \leq 1$

Fig. 3.3: Instantaneous evolution of the demand in the Riemann problem (incoming road).

Using the same approach for the outgoing road, we have:

$$
\begin{array}{ll}
\sigma\left(\rho_{2,0}\right)=\sigma\left(\hat{\rho}_{2}\right) & \text { if } 0 \leq \rho_{2,0} \leq \rho^{\mathrm{cr}}, \\
\sigma\left(\rho_{2,0}\right) \leq \sigma\left(\hat{\rho}_{2}\right) & \text { if } \rho^{\mathrm{cr}} \leq \rho_{2,0} \leq 1,
\end{array}
$$

In particular, if $0 \leq \rho_{2,0} \leq \rho^{\text {cr }}$ then $0 \leq \hat{\rho}_{2} \leq \rho^{\text {cr }}$ as well, and $\sigma\left(\rho_{2,0}\right)=f^{\max }=\sigma\left(\hat{\rho}_{2}\right)$, see Figure 3.4(c). Otherwise $\rho^{\text {cr }} \leq \rho_{2,0} \leq 1$, and either $\hat{\rho}_{2}=\rho_{2,0}$ or $\hat{\rho}_{2} \in\left[0, \tau\left(\rho_{2,0}\right)[\right.$. In the first case, $\sigma\left(\rho_{2,0}\right)=f\left(\rho_{2,0}\right)=f\left(\hat{\rho}_{2}\right)=\sigma\left(\hat{\rho}_{2}\right)$, see Figure 3.4(b) In the second case, $\sigma\left(\rho_{2,0}\right)=f\left(\rho_{2,0}\right) \leq f^{\max }=\sigma\left(\hat{\rho}_{2}\right)$, see Figure 3.4(a).

For the onramp, we consider two different cases, when the buffer is initially empty and when it is not. In both cases different situations can occur.

(L1) Initially empty buffer: $l(0)=0 \Rightarrow d\left(F_{\mathrm{in}}, l(0)\right)=\min \left(F_{\mathrm{in}}, \gamma_{\mathrm{r} 1}^{\max }\right)$.

(L1.1) Buffer increases: $l(0+)>0 \Rightarrow d\left(F_{\mathrm{in}}, l(0+)\right)=\gamma_{\mathrm{r} 1}^{\max }$.

If $d\left(F_{\text {in }}, l(0)\right)=F_{\text {in }}$, then $d\left(F_{\text {in }}, l(0)\right) \leq d\left(F_{\text {in }}, l(0+)\right)$.

If $d\left(F_{\mathrm{in}}, l(0)\right)=\gamma_{\mathrm{r} 1}^{\max }$, then $d\left(F_{\mathrm{in}}, l(0)\right)=d\left(F_{\mathrm{in}}, l(0+)\right)$.

(L1.2) Buffer remains empty: $l(0+)=0 \Rightarrow d\left(F_{\text {in }}, l(0+)\right)=\min \left(F_{\text {in }}, \gamma_{\mathrm{r} 1}^{\max }\right)$. Hence, $d\left(F_{\text {in }}, l(0)\right)=d\left(F_{\text {in }}, l(0+)\right)$.

(L2) Buffer initially not empty: $l(0)>0 \Rightarrow d\left(F_{\text {in }}, l(0)\right)=\gamma_{\mathrm{r} 1}^{\max }$.

(L2.1) Buffer grows (decreases) linearly: $0<l(0)<l(0+)(0<l(0+)<l(0)) \Rightarrow$ $d\left(F_{\mathrm{in}}, l(0+)\right)=\gamma_{\mathrm{r} 1}^{\max }$.

Hence, $d\left(F_{\text {in }}, l(0)\right)=d\left(F_{\text {in }}, l(0+)\right)$. 


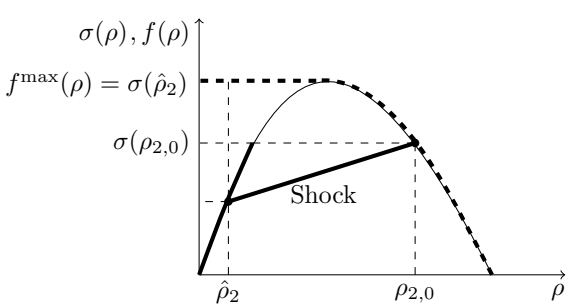

(a) $\rho^{\text {cr }} \leq \rho_{2,0} \leq 1$

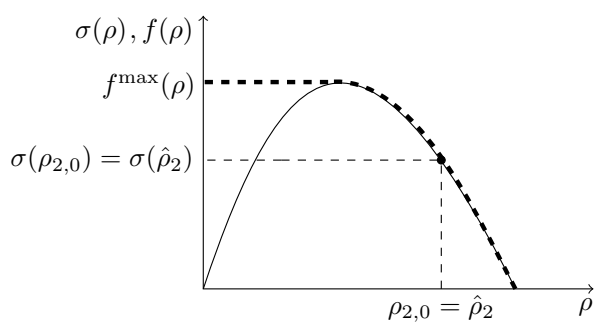

(b) $\rho_{2,0}=\hat{\rho}_{2}$

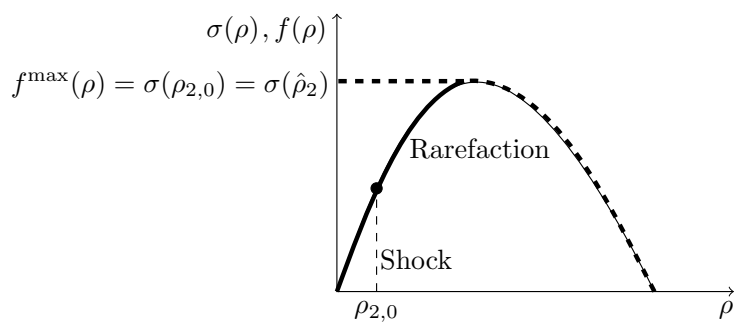

(c) $0 \leq \rho_{2,0} \leq \rho^{\mathrm{cr}}$

Fig. 3.4: Instantaneous evolution of the supply in the Riemann problem (outgoing road).

This concludes the proof.

Now we are ready to prove Theorem 3.2 .

Proof. Existence and uniqueness follow by construction of the Riemann Solver detailed at the beginning of this section.

In the following we will show the proof of the consistency of $\mathcal{R} \mathcal{S}_{l(t)}$.

Fix $t_{0} \geq 0$. If $\left(\rho_{1}\left(t_{0}, 0-\right), \rho_{2}\left(t_{0}, 0+\right)\right)$ is a solution of the Riemann Solver, corresponding to the same buffer value $l\left(t_{0}\right)$ we need to show that

$$
\mathcal{R S}_{l\left(t_{0}\right)}\left(\rho_{1}\left(t_{0}, 0-\right), \rho_{2}\left(t_{0}, 0+\right)\right)=\left(\rho_{1}\left(t_{0}, 0-\right), \rho_{2}\left(t_{0}, 0+\right)\right)
$$

Without loss of generality, we fix $t_{0}=0$ and we keep the same notation used in the proof of Lemma 3.3 .

We show that the optimal point in the feasible set $\Omega$, as defined in (3.1), resulting from the Riemann Solver does not change. From the results of Lemma 3.3 it is straightforward to say that the set $\Omega$ either increases between times $t=0$ and $t>0$ or does not change, see Figures 3.5 and 3.6. Now, we need to prove that the optimal point does not change. We treat the supply constrained and the demand limited cases separately. 


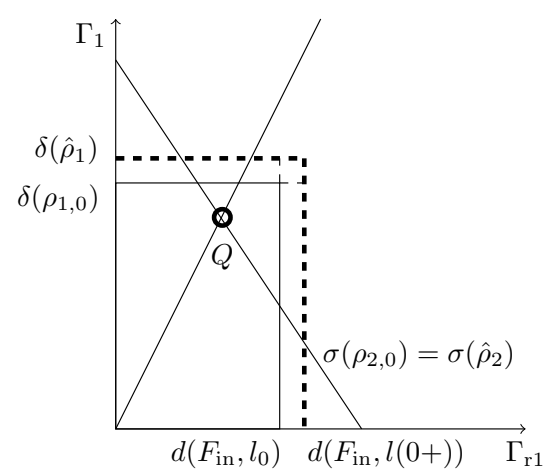

(a) $Q \in \Omega$

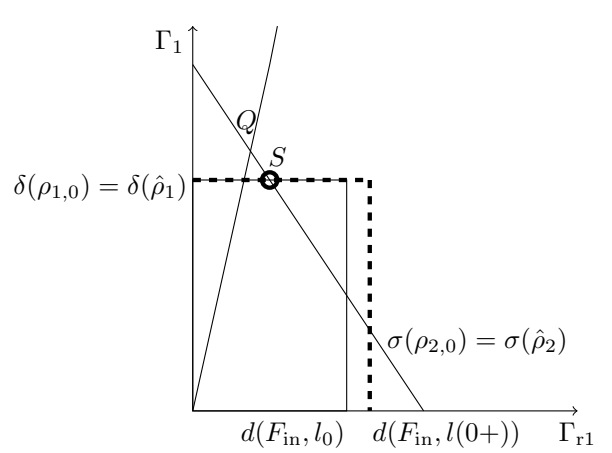

(b) $Q \notin \Omega, \hat{\Gamma}_{1}=\delta\left(\rho_{1,0}\right)$

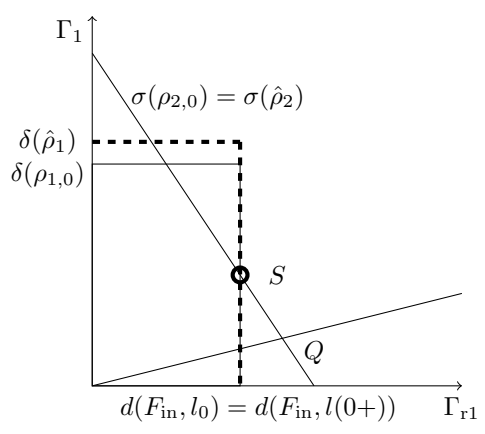

(c) $Q \notin \Omega, \hat{\Gamma}_{\mathrm{r} 1}(0)=d\left(F_{\text {in }}, l_{0}\right)$

Fig. 3.5: Supply constrained junction problems.

1. Supply constrained junction problem, see Figure 3.5

We assume that we are supply limited at $t=0$. In this case, by construction of the Riemann Solver, it holds $\rho_{2,0}=\hat{\rho}_{2}$ and hence $\sigma\left(\rho_{2,0}\right)=\sigma\left(\hat{\rho}_{2}\right)$. The priority line is fixed, the point of intersection $Q$ does not change.

(i) Optimal solution inside $\Omega$, see Figure 3.5)(a)

Since $Q$ is determined by the intersection of the two lines and $\Omega$ can only increase $\left(\delta\left(\rho_{1,0}\right) \leq \delta\left(\hat{\rho}_{1}\right), d\left(F_{\text {in }}, l(0)\right) \leq d\left(F_{\text {in }}, l(0+)\right)\right)$, we have

$$
\left(\hat{\rho}_{1}, \hat{\rho}_{2}\right)=\mathcal{R} \mathcal{S}_{l(0)}\left(\hat{\rho}_{1}, \hat{\rho}_{2}\right) .
$$

(ii) Optimal solution on the border of $\Omega, \hat{\Gamma}_{1}=\delta\left(\rho_{1,0}\right)$, see Figure 3.5 (b).

We have to prove that the result of the minimization problem (3.2) (the point $S$ in the figure) does not change. In this case, by construction of the Riemann Solver it holds $\rho_{1,0}=\hat{\rho}_{1}$. This yields $\delta\left(\rho_{1,0}\right)=\delta\left(\hat{\rho}_{1}\right)$ by (3.8). Since, $d\left(F_{\text {in }}, l(0)\right)$ can only increase according to the cases (L1) and (L2), it holds

$$
\left(\hat{\rho}_{1}, \hat{\rho}_{2}\right)=\mathcal{R} \mathcal{S}_{l(0)}\left(\hat{\rho}_{1}, \hat{\rho}_{2}\right) .
$$

(iii) Optimal solution on the border of $\Omega, \hat{\Gamma}_{\mathrm{r} 1}(0)=d\left(F_{\mathrm{in}}, l(0)\right)$, see Figure 3.5.|(c). For the onramp, the only case where the demand can increase is the case (L1.1). In this particular setting, if $d\left(F_{\text {in }}, l(0)\right)=F_{\text {in }}$ it holds $\gamma_{r 1}(0)=F_{\text {in }}$ and $F_{\text {in }} \leq \gamma_{\mathrm{r} 1}^{\max }$. When the 
buffer increases we have $\gamma_{\mathrm{r} 1}(0+)=d\left(F_{\mathrm{in}}, l(0+)\right)=\gamma_{\mathrm{r} 1}^{\max }$, which implies $\gamma_{\mathrm{r} 1}^{\max } \leq F_{\mathrm{in}}$. Hence, $F_{\mathrm{in}}=\gamma_{\mathrm{r} 1}^{\max }$ and $d\left(F_{\mathrm{in}}, l(0)\right)=d\left(F_{\mathrm{in}}, l(0+)\right)$. The mainline demand can only increase. Hence,

$$
\left(\hat{\rho}_{1}, \hat{\rho}_{2}\right)=\mathcal{R} \mathcal{S}_{l(0)}\left(\hat{\rho}_{1}, \hat{\rho}_{2}\right) .
$$

2. Demand constrained junction problem, see Figure 3.6 .

$\Omega=\Omega\left(\rho_{1,0}, l(0)\right)=\Omega\left(\hat{\rho}_{1}, l(0)\right)$. In fact, $\rho_{1,0}=\hat{\rho}_{1}$ and for the onramp it holds $\gamma_{\mathrm{r} 1}^{\max }=F_{\mathrm{in}}$ (as in the previous point), and this yields $\delta\left(\rho_{1,0}\right)=\delta\left(\hat{\rho}_{1}\right)$ and $d\left(F_{\mathrm{in}}, l(0)\right)=$ $d\left(F_{\mathrm{in}}, l(0+)\right)$ by 3.8 and (L1) and (L2). The supply can only increase by 3.9 . Hence,

$$
\left(\hat{\rho}_{1}, \hat{\rho}_{2}\right)=\mathcal{R} \mathcal{S}_{l(0)}\left(\hat{\rho}_{1}, \hat{\rho}_{2}\right)
$$

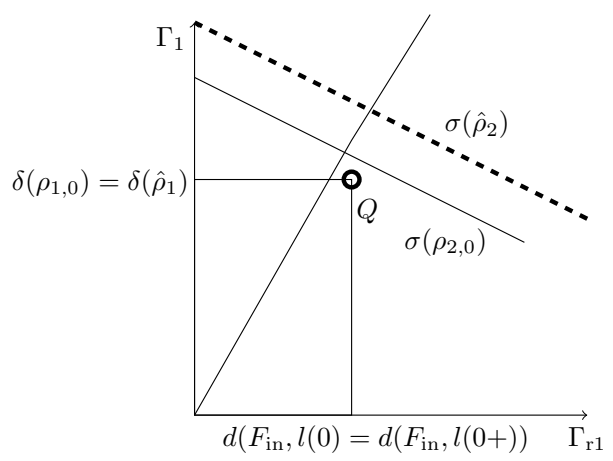

Fig. 3.6: Demand constrained junction problem.

Moreover, the limiting side of $\Omega$ does not change, i.e., it is not possible to pass from a demand constrained junction problem to a supply constrained one and viceversa. This follows for the fact that $\sigma\left(\rho_{2,0}\right)=\sigma\left(\hat{\rho}_{2}\right)$ when we have a supply constrained junction problem, Figure 3.5 and $d\left(F_{\mathrm{in}}, l(0)\right)=d\left(F_{\mathrm{in}}, l(0+)\right), \delta\left(\rho_{1,0}\right)=\delta\left(\hat{\rho}_{1}\right)$ when we have a demand constrained junction problem, Figure 3.6.

This concludes the proof.

REMARK 3. The proposed model is a variant of the junction model considered in [4] in the $2 \times 2$ case. In [4], the traffic distribution across the junction is given by a distribution matrix $A$, subject to technical conditions that ensure uniqueness of the solution. In our case, since we suppose that no flux from the onramp is directed into the offramp, the distribution matrix would look as: $A=\left(\begin{array}{cc}1-\beta & 1 \\ \beta & 0\end{array}\right)$. Clearly, as the offramp gets more congestioned, $\beta$ decreases. If we solve the model proposed in [4] using this distribution matrix, there can be cases in which the solution gives zero onramp flux, see Figure 3. I( a). This is due to the choice of maximizing the flow throughout the junction. In fact, in this way, the model tends to satisfy the mainline demand before the onramp one. This does not reflect what happens in reality, since the demand allocation depends on the number of lanes available for each inflow, for details see [13]. Hence, we propose a model that fix this issue balancing the flux between the two incoming roads by the introduction of a right-of-way parameter. In particular, the priority coefficient keeps the maximization point far from the axis, avoiding blocking, see Figure 3. W(b). 


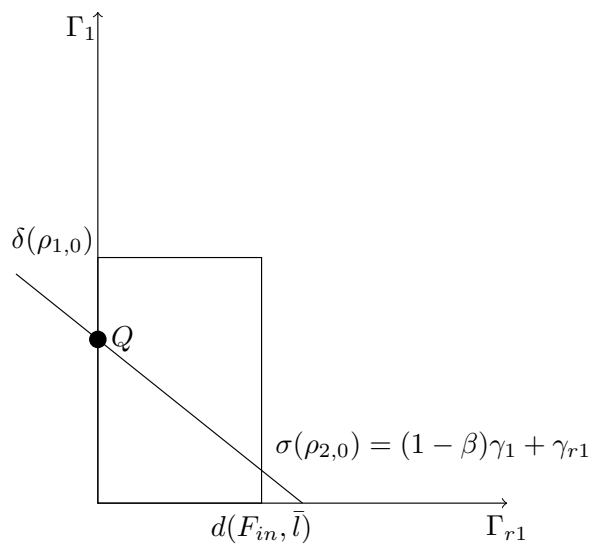

(a)

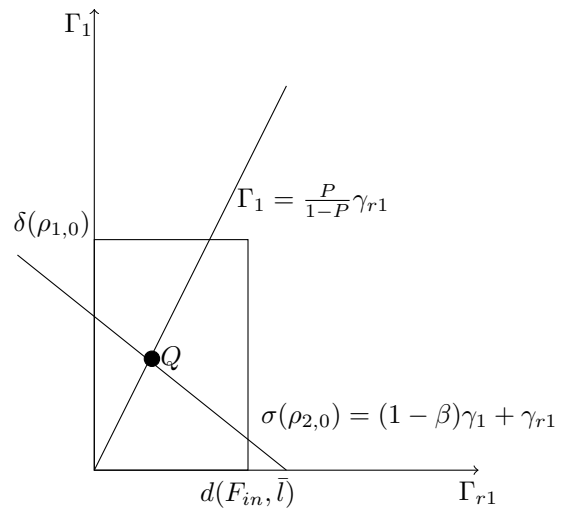

(b)

Fig. 3.7: Comparison between Coclite-Garavello-Piccoli model 4](left) and our model (right).

4. Numerical Approximation. In order to find approximate solutions, we adapt the classical Godunov scheme to the problem, with some adjustment due to the presence of the buffer.

We define a numerical grid in $(0, T) \times \mathbb{R}$ using the following notation:

(i) $\Delta x$ is the fixed space grid size;

(ii) $\Delta t^{n}$ is the non-uniform time grid size given by the CFL condition;

(iii) $\left(t^{n}, x_{j}\right)=\left(t^{n-1}+\Delta t^{n}, j \Delta x\right)$ for $n \in \mathbb{N}$ and $j \in \mathbb{Z}$ are the grid points.

For a function $v$ defined on the grid we write $v_{j}^{n}=v\left(t^{n}, x_{j}\right)$ for $j, n$ varying on a subset of $\mathbb{Z}$ and $\mathbb{N}$ respectively. We also use the notation $u_{j}^{n}$ for $u\left(t^{n}, x_{j}\right)$ when $u$ is a continuous function on the $(t, x)$ plane.

4.1. Godunov Scheme. The Godunov scheme as introduced in [10] is based on exact solutions to Riemann problems. The main idea of this method is to approximate the initial datum by a piecewise constant function, then the corresponding Riemann problems are solved exactly and a global solution is simply obtained by piecing them together. Finally, one takes the mean on the cell and proceeds by induction. Consider the hyperbolic problem with initial data

$$
\begin{array}{cl}
\partial_{t} u+\partial_{x} f(u)=0 & x \in \mathbb{R}, t \in[0, T], \\
u(0, x)=u_{0}(x) & x \in \mathbb{R} .
\end{array}
$$

A solution of the problem is constructed taking a piecewise constant approximation of the initial data:

$$
v_{j}^{0}=\frac{1}{\Delta x} \int_{x_{j-1 / 2}}^{x_{j+1 / 2}} u_{0}(x) d x \quad j \in \mathbb{Z},
$$

and defining $v_{j}^{n}$ recursively starting from $v_{j}^{0}$, as follows.

$$
v_{j}^{n+1}=\frac{1}{\Delta x} \int_{x_{j-1 / 2}}^{x_{j+1 / 2}} v^{\Delta}\left(t^{n+1}, x\right) d x .
$$


Under the CFL condition

$$
\Delta t^{n} \max _{j \in \mathbb{Z}}\left|\lambda_{j+\frac{1}{2}}^{n}\right| \leq \frac{1}{2} \Delta x
$$

the waves generated by different Riemann problems do not interact. Above, $\lambda_{j+\frac{1}{2}}^{n}$ is the wave speed of the Riemann problem solution at the interface $x_{j+\frac{1}{2}}$ at time $t^{n}$. Under the condition 4.5 the scheme can be written as

$$
v_{j}^{n+1}=v_{j}^{n}-\frac{\Delta t^{n}}{\Delta x}\left(g\left(v_{j}^{n}, v_{j+1}^{n}\right)-g\left(v_{j-1}^{n}, v_{j}^{n}\right)\right),
$$

where numerical flux $g$ takes in general the following expression:

$$
g(u, v)= \begin{cases}\min _{z \in[u, v]} f(z) & \text { if } u \leq v \\ \max _{z \in[v, u]} f(z) & \text { if } v \leq u\end{cases}
$$

4.2. Boundary conditions and conditions at the junctions. Here we impose the boundary conditions for the incoming and the outgoing roads at the endpoint not connected to the junction. We also assign boundary conditions at the endpoints of the roads connected to the junction. In both cases we will use the classical approach for road networks as introduced in [3].

Boundary conditions. Each road is divided in $J+1$ cells numbered from 0 to $J$. For the incoming road, in practice, we proceed defining

$$
v_{0}^{n+1}=v_{0}^{n}-\frac{\Delta t^{n}}{\Delta x}\left(g\left(v_{0}^{n}, v_{1}^{n}\right)-f\left(v_{0}^{n}\right)\right),
$$

where $f\left(v_{0}^{n}\right)$ is the value of the flux at the boundary.

An outgoing boundary can be treated analogously,

$$
v_{J}^{n+1}=v_{J}^{n}-\frac{\Delta t^{n}}{\Delta x}\left(f\left(v_{J}^{n}\right)-g\left(v_{J-1}^{n}, v_{J}^{n}\right)\right),
$$

with $f\left(v_{J}^{n}\right)$ the outgoing flux.

Since we are dealing with Riemann problems at the junction, the formulation of absorbing boundary conditions is equivalent to the one with the ghost cells which is common in literature, see for details [3, 4, 23].

Conditions at the Junction. For $I_{1}$, that is connected at the junction at the right endpoint, we set

$$
v_{J}^{n+1}=v_{J}^{n}-\frac{\Delta t^{n}}{\Delta x}\left(\hat{\Gamma}_{1}-g\left(v_{J-1}^{n}, v_{J}^{n}\right)\right),
$$

while for the outgoing road, connected at the junction at the left endpoint, we have

$$
v_{0}^{n+1}=v_{0}^{n}-\frac{\Delta t^{n}}{\Delta x}\left(g\left(v_{0}^{n}, v_{1}^{n}\right)-\hat{\Gamma}_{2}\right),
$$

where $\hat{\Gamma}_{1}$ and $\hat{\Gamma}_{2}$ are the maximized fluxes computed in $\$ 3$.

REMARK 4. For Godunov scheme there is no need to invert the flux $f$ to compute the corresponding density in the scheme, as the Godunov flux coincides with Riemann flux. In this case it suffices to insert the computed maximized fluxes directly into the scheme. 
4.3. ODE Treatment. Let us consider now the buffer modeled by 2.2 on the onramp. At each time step $t^{n}=t^{n-1}+\Delta t^{n}$ we compute the new value of the queue length according to two possible cases, with Euler first order integration.

1. If $F_{\text {in }}\left(t^{n}\right)<\hat{\Gamma}_{\text {r1 }}$

$$
l^{n+1}= \begin{cases}l^{n}+\left(F_{\mathrm{in}}\left(t^{n}\right)-\hat{\Gamma}_{\mathrm{r} 1}\right) \Delta t^{n} & \text { for } t^{n+1}<\bar{t} \\ 0 & \text { otherwise }\end{cases}
$$

2. If $F_{\text {in }}\left(t^{n}\right) \geq \hat{\Gamma}_{\text {r1 }}$

$$
l^{n+1}=l^{n}+\left(F_{\text {in }}\left(t^{n}\right)-\hat{\Gamma}_{\mathrm{r} 1}\right) \Delta t^{n} .
$$

Above, $\hat{\Gamma}_{\mathrm{r} 1}$ is the maximized flux described in $\$ 3, F_{\text {in }}\left(t^{n}\right)$ is the flux entering the onramp at $t^{n}$ given by

$$
F_{\text {in }}\left(t^{n}\right)=\frac{1}{\Delta t^{n}} \int_{t^{n}}^{t^{n+1}} F_{\text {in }}(t) d t,
$$

and $\bar{t}$ is the time at which the buffer empties. We can calculate the time at which the buffer can empty for each time step $\Delta t^{n}$ :

$$
\bar{t}=-\frac{l^{n}}{F_{\mathrm{in}}\left(t^{n}\right)-\hat{\Gamma}_{\mathrm{r} 1}}+t^{n} .
$$

4.4. Modified Godunov Scheme. Godunov scheme cannot be applied as it is when the buffer empties, because the solution could potentially not be self-similar. If the buffer empties, at some time step $\Delta t^{n}$, we might have multiple shocks at the junction. In this case we divide the time step $\Delta t^{n}=\left(t^{n}, t^{n+1}\right)$ in two sub-intervals $\Delta t_{a}=\left(t^{n}, \bar{t}\right)$ and $\Delta t_{b}=\left(\bar{t}, t^{n+1}\right)$, as in Figure 4.1. with $\bar{t}$ being defined in 4.8). Then, we solve in one time step two different Riemann Problems at the junction. For $\Delta t_{a}$ we solve the classical Godunov scheme. For the $\Delta t_{b}$ we solve a new Riemann Problem at the junction in which the value of the queue length is $l=0$. The junction conditions are

$$
\begin{gathered}
v_{J}^{n+1}=v_{J}^{\bar{t}}-\frac{\Delta t_{b}}{\Delta x}\left(\hat{\Gamma}_{1}^{\bar{t}}-g\left(v_{J-1}^{n}, v_{J}^{\bar{t}}\right)\right), \\
v_{0}^{n+1}=v_{0}^{\bar{t}}-\frac{\Delta t_{b}}{\Delta x}\left(g\left(v_{0}^{\bar{t}}, v_{1}^{n}\right)-\hat{\Gamma}_{2}^{\bar{t}}\right),
\end{gathered}
$$

where with the superscript $\bar{t}$ we indicate the value computed at $t=\bar{t}$ in the previous time step $\Delta t_{a}$.

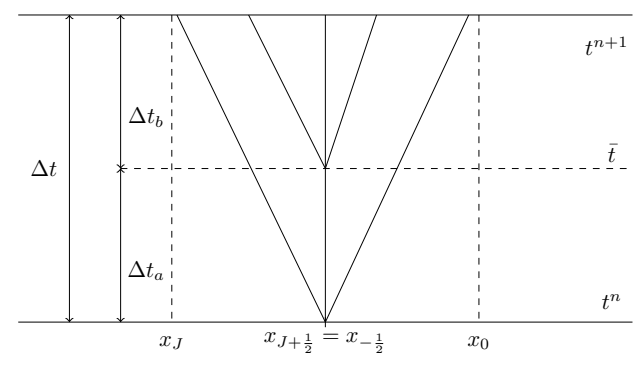

Fig. 4.1: Junction in the case of emptying buffer. 
5. Numerical Results. For illustration, we choose a concave fundamental diagram with the following flux function:

$$
f(\rho)=V_{\max } \rho(1-\rho)
$$

where $V_{\max }$ is the maximal velocity of the traffic flow. In this case the Godunov numerical flux is given by

$$
g(u, v)= \begin{cases}\min (f(u), f(v)) & \text { if } u \leq v, \\ f(u) & \text { if } v<u<\rho^{\mathrm{cr}}, \\ f^{\max } & \text { if } v<\rho^{\mathrm{cr}}<u, \\ f(v) & \text { if } \rho^{\mathrm{cr}}<v<u\end{cases}
$$

In this section we present some numerical tests performed with the scheme previously described. We introduce the formal order of convergence $\mu$ of a numerical method

$$
\mu=\frac{\ln \left(\mathrm{TOT}_{\mathrm{err}}\right)}{\ln (d x)}
$$

where the $\mathbf{L}^{\mathbf{1}}$-norm error is given by

$$
\mathrm{TOT}_{\mathrm{err}}=\sum_{i=1}^{2}\left\|u_{e}^{i}-u_{c}^{i}\right\|_{\mathbf{L}^{1}}
$$

where $u_{e}^{i}$ and $u_{c}^{i}$ are the exact solution and the computed solution in each road, respectively. We show some numerical results obtained applying Godunov scheme to problem 2.3. Tables 5.1 and 5.2 provide the values of the $\mathbf{L}^{1}$-error 5.3 and the order of convergence 5.2 . Here we deal with a mainline of length 8 parametrized by the interval $[-4,4]$ with the node placed at $x=0$, such that $I_{1}=[-4,0]$ and $I_{2}=\left[0,4\right.$.] In all the simulations we fix $V_{\max }=1, P=0.7, \beta=0.2, \gamma_{\max }=0.5$, $l_{0}=0.2$ and $F_{\text {in }}=0.05$.

1. Case I: We consider the following initial data

$$
\rho_{1}(0, x)=0.6, \quad \rho_{2}(0, x)=0 .
$$

The values of the initial conditions creates a shock on the incoming mainline and a rarefaction on the outgoing one. After a time $t=5.3$ we can see the rarefaction caused by the buffer that empties in the incoming mainline $I_{1}$, as illustrated in Figure 5.1 . 

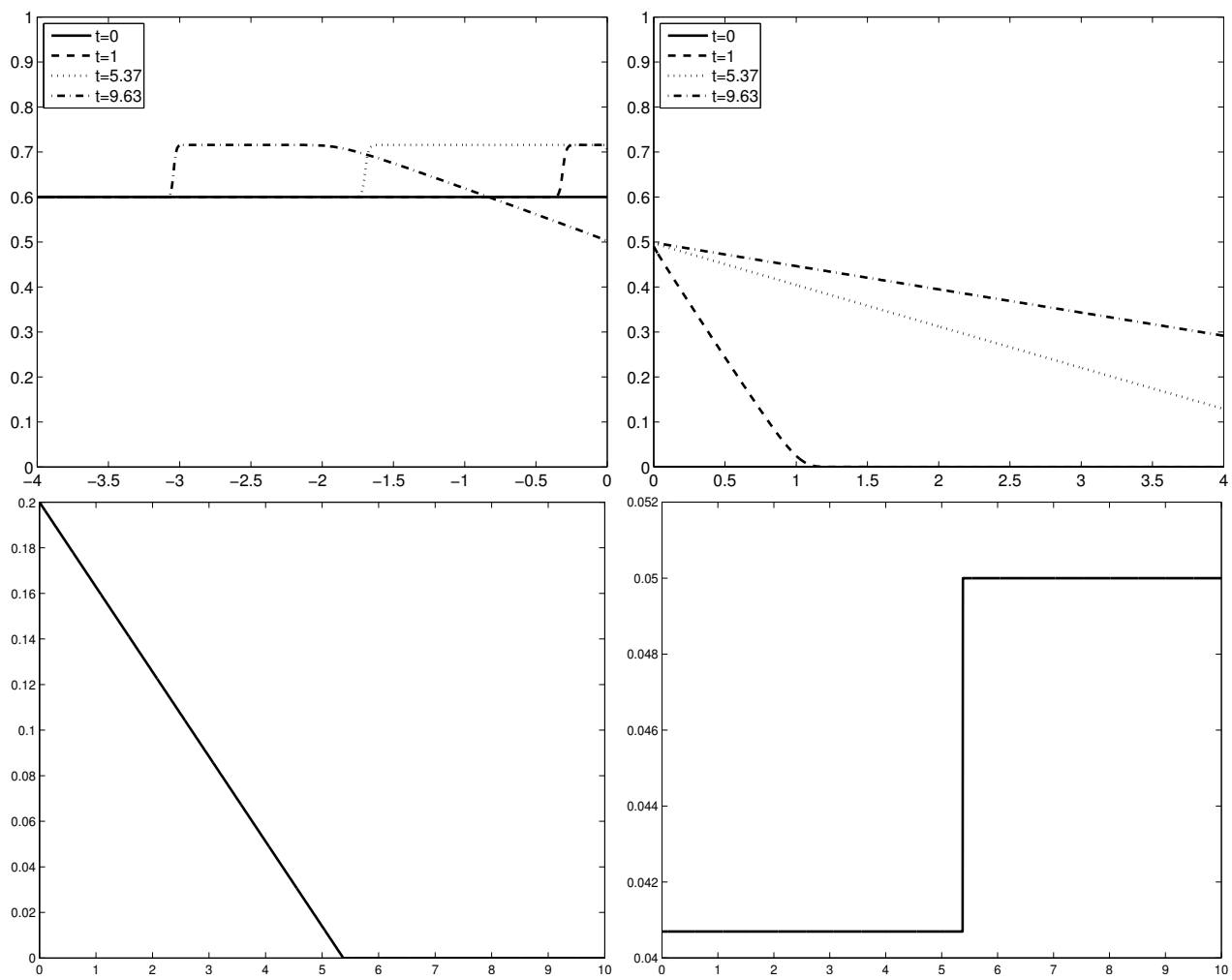

Fig. 5.1: Evolution in time of the density in the incoming mainline (above, left), on the outgoing mainline (above, right) and evolution of the flux in the onramp (bottom, left) and in the offramp (bottom, right), corresponding to initial data (5.4) and a space step discretization $\Delta x=0.01$.

Table 5.1 collects the values of the $\mathbf{L}^{\mathbf{1}}$-error and of the order of convergence at time $T=10$.

\begin{tabular}{|c|c|c|}
\hline$d x$ & $\mathbf{L}^{\mathbf{1}}$-error & $\mu$ \\
\hline 0.02 & $3.69 \cdot 10^{-2}$ & 0.8432 \\
\hline 0.01 & $1.49 \cdot 10^{-2}$ & 0.9133 \\
\hline 0.005 & $7.21 \cdot 10^{-3}$ & 0.9322 \\
\hline 0.002 & $1.10 \cdot 10^{-3}$ & 1.0962 \\
\hline 0.001 & $2.23 \cdot 10^{-4}$ & 1.2170 \\
\hline
\end{tabular}

Table 5.1: Errors and order of convergence for the Godunov scheme at time $T=10$, corresponding to initial data (5.4).

2. Case II: We consider the following initial data

$$
\rho_{1}(0, x)=0.1, \quad \rho_{2}(0, x)=0.6 .
$$

In this case, the values of the initial conditions are chosen such that the wave produced by the buffer that empties can be seen in the outgoing mainline. In particular, in this 
case no waves are generated at initial time. The only wave generated is a shock which appear once that the buffer empties at time $t=1.53$, as shown in Figure 5.2 Table 5.2 reports the $\mathbf{L}^{\mathbf{1}}$-error and the order of convergence.
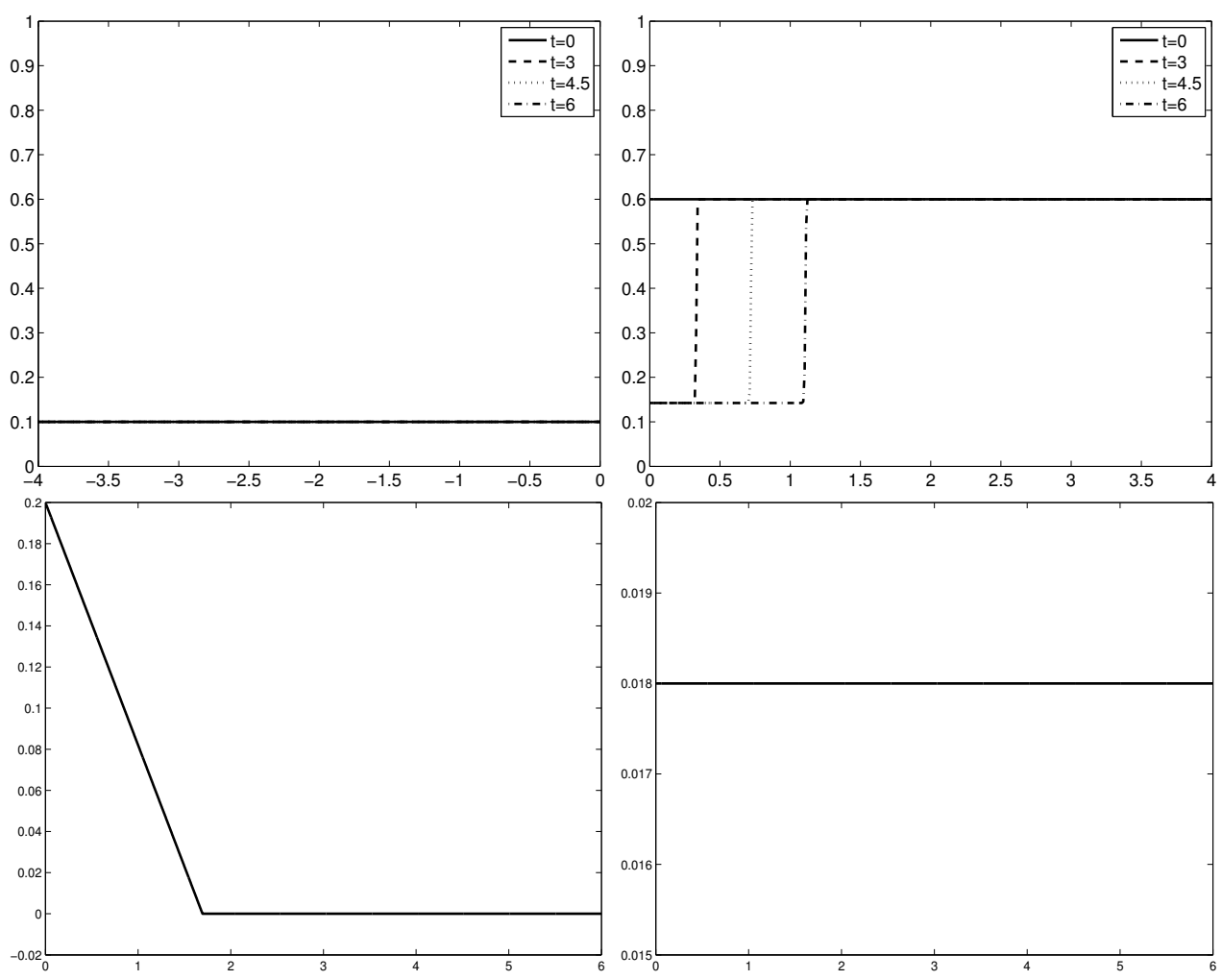

Fig. 5.2: Evolution in time of the density in the incoming mainline (above, left), on the outgoing mainline (above, right) and evolution of the flux in the onramp (bottom, left) and in the offramp (bottom, right), corresponding to initial data 5.5 and a space step discretization $\Delta x=0.01$.

\begin{tabular}{|c|c|c|}
\hline$d x$ & $\mathbf{L}^{\mathbf{1}}$-error & $\mu$ \\
\hline 0.02 & $1.70 \cdot 10^{-2}$ & 1.0464 \\
\hline 0.01 & $1.67 \cdot 10^{-2}$ & 0.8890 \\
\hline 0.005 & $1.44 \cdot 10^{-2}$ & 0.8066 \\
\hline 0.002 & $9.39 \cdot 10^{-3}$ & 0.9878 \\
\hline 0.001 & $3.57 \cdot 10^{-4}$ & 1.2474 \\
\hline
\end{tabular}

Table 5.2: Errors and order of convergence for the Godunov scheme at time $T=3$, corresponding to initial data (5.5).

6. Conclusions. This article introduces a model of a $2 \times 2$ junction with an onramp and an offramp. The onramp is modeled by an ODE which represent a vertical buffer. This way of handling boundary conditions makes possible not to lose 
flow information. The junction flow distribution is solved through a $L P$-optimization problem, which maximizes the flow in the outgoing mainline. Moreover, a right-ofway parameter is introduced to ensure the uniqueness of the solution and a good representation of field experiences. The model is solved numerically using a modified Godunov scheme that takes into account the waves that can be produced when the buffer empties. Some numerical tests are presented to show the stability and accuracy of the scheme. Moreover, from the analysis of the $\mathbf{L}^{\mathbf{1}}$ error, the convergence of the scheme is demonstrated numerically.

\section{REFERENCES}

[1] C. Bardos, A. Y. Le Roux, And J.-C. NÉdÉlec, First order quasilinear equations with boundary conditions, Comm. Partial Differential Equations, 4 (1979), pp. 1017-1034.

[2] A. Bressan, Hyperbolic systems of conservation laws, vol. 20 of Oxford Lecture Series in Mathematics and its Applications, Oxford University Press, Oxford, 2000. The one-dimensional Cauchy problem.

[3] G. Bretti, R. Natalini, and B. Piccoli, Numerical approximations of a traffic flow model on networks, Networks and Heterogeneous Media, 1 (2006), p. 57.

[4] G.M. Coclite, M. Garavello, and B. Piccoli, Traffic flow on a road network, SiAM J. Math. Anal., 36(6) (2005), pp. 1862-1886.

[5] R. M. Colombo, P. Goatin, And B. Piccoli, Road network with phase transition, Journal of Hyperbolic Differential Equations, 07 (2010), pp. 85-106.

[6] C.M. Dafermos, Hyperbolic Conservation Laws in Continuum Physics, Springer, 2009.

[7] M. Garavello and P. Goatin, The Cauchy problem at a node with buffer, Discrete Contin. Dyn. Syst. Ser. A, 32(6) (2012), pp. 1915-1938.

[8] M. Garavello and B. Piccoli, Traffic flow on a road network using the Aw-Rascle model, Comm. Partial Differential Equations, 31 (2006), pp. 243-275.

[9] - Traffic Flow on Networks: Conservation Laws Model, AIMS Series on Applied Mathematics, American Institute of Mathematical Sciences, 2006.

[10] S.K. Godunov, A finite difference method for the numerical computation of discontinuous solutions of the equations of fluid dynamics, Matematicheskii Sbornik, 47 (1959), pp. 271290.

[11] M. Herty, J. Lebacque, and S. Moutari, A novel model for intersections of vehicular traffic flow, Netw. Heterog. Media, 4 (2009), pp. 813-826.

[12] M. Herty, S. Moutari, and Rascle A., Optimization criteria for modelling intersections of vehicular traffic flow, Netw. Heterog. Media, 1 (2006), pp. 275-294.

[13] W. Krichene, M.L. Delle Monache, J. Reilly, S. Samaranayake, P. Goatin, and A.M. BAYEN, A continuous model for traffic networks. in preparation.

[14] S. N. KRUŽHKOV, First order quasilinear equations with several independent variables., Mat. Sb. (N.S.), 81 (123) (1970), pp. 228-255.

[15] P. LE FLOCH, Explicit formula for scalar non-linear conservation laws with boundary condition, Mathematical Methods in the Applied Sciences, 10 (1988), pp. 265-287.

[16] R.J. LeVeque, Numerical Methods for Conservation Laws, Lectures in mathematics ETH Zürich, Birkhäuser, 1992.

[17] _ Finite Volume Methods for Hyperbolic Problems, Cambridge Texts in Applied Mathematics, Cambridge University Press, 2002.

[18] M. J. Lighthill and B. Witham, On kinematic waves. ii. a theory of traffic flow on long crowded roads, Proc. Roy. Soc. London Ser. A, 229 (1955), pp. 317-346.

[19] A. Muralidharan, G. Dervisoglu, and Horowitz R., Freeway traffic flow simulation using the link node cell transmission model, in American Control Conference, 2009.

[20] A. Muralidharan and Horowitz R., Optimal control of freeway networks based on the link node cell transmission model, in American Control Conference, 2012. pre-print.

[21] P. I. Richards, Shock waves on the highway, Operations Research, 4 (1956), pp. 42-51.

[22] I. S. Strub And A. Bayen, Weak formulation of boundary conditions for scalar conservation laws: An application to highway traffic modelling, International Journal of Robust and Nonlinear Control, 16 (2006), pp. 733-748.

[23] D. Work, S. Blandin, O.-P. Tossavainen, B. Piccoli, and A. M. Bayen, A distributed highway velocity model for traffic state reconstruction, Applied Mathematics Research eXpress (AMRX), 1 (2010), pp. 1-35. 